\title{
EDITORIAL
}

\section{La investigación en salud}

\section{The investigation in health}

Ruby Elena Muñoz Baldiris ${ }^{1}$

La orientación de la investigación en salud, remontada a la creación por el Departamento Administrativo de Ciencia, Tecnología e Innovación - COLCIENCIAS -, del Programa Nacional de Ciencia y Tecnología de la Salud, se sintoniza con los lineamientos de la Política Nacional de Fomento a la Investigación y la Innovación "Colombia Construye y Siembra Futuro"; y apunta a que la misma, produzca conocimiento que aporte al mejoramiento de la salud, de las condiciones de vida, de la equidad, del desarrollo social y por tanto del desarrollo económico de las regiones y poblaciones ${ }^{2}$.

Se asume entonces, desde los lineamientos de la Política Nacional de Fomento a la Investigación y la Innovación, que la investigación en salud puede agregar valor a las decisiones y optimizar la solución de los problemas que aquejan a una sociedad; al ser considerada esta, como estrategia fundamental para aportar al Estado Colombiano, a las instituciones y al conjunto de la sociedad, entre otros, conocimiento y comprensión de los problemas de salud que persisten y de los emergentes; entendimiento de los aspectos económicos, sociales y de otros factores determinantes de la salud, así como el fortalecimiento de la línea base para diseñar programas y servicios eficaces y efectivos.

Desde la anterior perspectiva, ha surgido la necesidad de priorizar la investigación en salud en nuestras condiciones, en el nivel regional y nacional. Así, el Programa Nacional de Ciencia y Tecnología de la Salud, plantea, para orientar la investigación en estos niveles, unas líneas de acción: investigación básica y biomédica, investigación clínica e investigación en salud pública. Pero es esto suficiente?, creo que no, pues la construcción de agendas de prioridades de investigación en salud, desde un punto de vista general, se constituye en proceso político que requiere consensos, en la medida que involucra diferentes actores, con experiencias y lenguajes diferentes, tanto en investigación como en salud.

En este orden de ideas, para facilitar los fines del desarrollo de la investigación en salud, en el sentido de generarse conocimiento para mejorar en esta área; creo debe existir desde las Agendas Departamentales de Ciencia, Tecnología e Innovación una definición de ejes

\footnotetext{
${ }^{1}$ MSc en Educación con Énfasis en Gerencia Educativa, Especialista en Epidemiología, Programa de Instrumentación Quirúrgica, Corporación Universitaria Rafael Núñez.

Correspondencia: ruby.munoz@curnvirtual.edu.co

2 Tomado de http://www.colciencias.gov.co/programa_estrategia/tecnolog-e-innovaci-n-en-salud
} 
estratégicos y apuestas en este sentido; al tiempo que acogiéndose la importancia de la investigación en salud para el bienestar social, contemplarse la evaluación del impacto de la investigación que se desarrolla en este campo, desde unos nuevos indicadores, para el caso, desde indicadores orientados a establecer el impacto social que la misma genera. 